\title{
Über die Function des Musculus Cramptonianus.
}

\author{
Von Prof. Sigm. Exmer, \\ Assistenten am physiologischen Institute. \\ (Mit 1 Tatel und I Holzschnitt.)
}

Der Musculus Crampromiunus ist bekanntlich ein im Ange der Vögel und einiger Reptilien nachgewiesener ringförmiger Muskel, dessen Fasern an der Innenwand des Scleralringes entspringen, und nach rorne verlaufend, sich an die innere Lamelle der Hornhaut ansetzen. Fig. 1 zeigt einen Durehschnitt durch das Ange des Uhu nach Brỉeke. ${ }^{1}$ Der dunkle Antheil der mit a bezeiehneten Stelle entspreehend, ist der in Rede stehende Muskel. Die durch ein Rechteck eingerahmte Stelle der Fig. 1 habe ich in Fig. 2 anf Grund eines anatomischen Präparates bei Lmpenvergrösserung wiedergegeben. Hier ist der Muskel punktirt. Bei vielen Vögeln findet sich der Museulus tensor eloreoideae in nemenswerther Entfernung von diesem Crampto n'schen Muskel, z. B. beim Ulin, wo er in Fig. 1 bei / angedeutet ist. Er ist stets dadureh charakterisirt, dass seine Muskelfasern vorne mit der Selera, beritglieh ihrem Knoehenring; hinten mit der Choreoidea verbunden sind. Bei anderen Vögehn aber riicken die beiden Muskeln einander näher, sowie dies in Fig. 3 ron einer Tanbe gezeiehnet ist. Die Fasern, welehe hier vom Periost des Knoehenringes entspringen und nach vorne verlanfen, gehören dom $\boldsymbol{~} \boldsymbol{H}$. Crrmptomirmus an, die, welehe vom Periost entspringen und nach hinten verlaufen, bilden den Tensor choreoideae. Bei der Taube und bei anderen Vögeh kommt zn diesen beiden Muskeh noch ein dritter hinzu. Wir wollen ihn mit L, enckart² die Miiller'sche

1 M ïll el's Arch. f. Anat. 4. Physiol. 1s4\%, pag. 370, 'I’at. XI.

z liräfe und Sämisch. Hanrlunch r. Ophthalmolog. 1. 2. pag. 231. 
Portion nennen. Er besteht aus Muskelfasern, welehe sich, wie die des Crampton'schen Muskels an der inneren Lamelle der Cornea einerseits, und wie die des Tensor choreoideae an der Choreoidea andererseits ansetzen. Sie haben also nirgends einen fixen Punkt. In meincr Zeichnung ist diese Müller'sche Portion zweibäuchig, wie man das sehr läufig sieht. Die mittlere Verdiinnung schafft fiir einen mächtigen Nervenstamm ( $n$ ) Platz, der zwisehen die Muskeln eingebettet ist. Die Zeichnung, welche Lenckart rom Truthahn gibt, ${ }^{1}$ zeigt die Mïller'sche Portion ohne diese Bäuche; ähulich die Königsteins, ${ }^{2}$ die dem Hulm entnommen ist und bei welcher auch zwischen den Muskelfasern der grosse Nervenstamm zu sehen ist. ${ }^{3}$

Was die Function dieser drei Fasergruppen anbelangt, so sind jetzt wohl alle Stimmen dariiber einig, dass der Tensor choreoideae, wie dies B r ü cke schon im Namen ausgedrickt hat, die Choreoidea um den Glaskörper anspannt. Er bewirkt in der bekannten Weise eine Accommodation fiur die Nähe. Dem gegeniiber ist iiber die Funetion der Mi il er'schen Portion noch nicht einmal eine Dentung ernstlich versucht worden, und über die des C'r a m p to n'schen Mnskels gehen die Anschanungen sehr' erheblich auseinander.

Crampton ${ }^{4}$ selbst war der Meinung, der von ilım entdeckte Muskel miisse bei seiner Contraetion die Cornea abflachen und demnach das Auge für die Ferne aceommodiren.

Brii ek e war der gegentheiligen Anschanng. Er wies nach, dass dic Hornhant des Vogels sieh in ilnen peripheren Theilen in "zwei Limellen spalten laisst, class diese in "der Gegend der Axe so mit einander verschnolyen sind, dass man ilure beiderseitige Grenze nieht auffinden kann; je weiter nan sich von der Axe entferut, un so weniger imnig wird der Zusanmmenhang, nud naeh dem Rande zu weichen beide Lamellen förmlich von einauder und lassen zwischen sich cine lockere, bindegewebentige Faserschicht eindringen, welche erlanbt, dass sie hier um ein Weniges

1 Ebenda.

- v. Frate's Aleh. f. Ophthalmolog. XXVI. 2, pag. 139. 'l'at. V, Fig. 1.

3 Eine andere Zeichnng desselben Autors, der 'Taube angehörig, zeigt dis: Verhältnisse iilndich wie meine \%eichnung.

1 (tilhert's Amalon, Bel, 4!, pang. 278 cit. wach Brifoke l, e: 
an einander verschoben werden können. Nummchr geht die äussere Lamelle in die Sclerotica iibcr und befestigt sich als solche an den vorderen Rand des Knochcnringes, inden sie eins wird mit der festen fibrösen Hant, welche den letzteren bekleidet. An die innere Lamelle dagegen sctzt sich der Crampton'sche Muskel". (Vergl. Fig.1, 2 und 3.) Auf Grund dieser anatomischen und hier nicht zu erörterıder physikalischer Verhältnisse erwartet Brïckc, dass die Contraction des Muskels den Kriimmungshalbmesser der Cornea rerkleinere, nnd dadurch das Ange fïr die Nähe accommodirc.

Cramer, der iibrigens cine unzweifelhaft unrichtige Anschanng vom anatomischen Verhalten des Crampton'schen Muskels liat, indem er ilın nicht an der Hornhaut, sonder'n an der Choreoidca, da, wo nach innen dic Ciliarfortsătze sitzcn, enden lässt, ' priifte Briicke's Auschauung experimentell und fand, dass elektrische Reizung des Anges auf die Hornbautkriimmung keinerlci Einfluss hat. ${ }^{2}$ Endlich hebt Königstcin ${ }^{3}$ hervor, dass der Crampton'sche Muskel und der Sphincter pupillae durch ihre vereinigte Wirkung indircet die Wände des Canalis Schlemmii von einander abheben und denselben dadurch öffncn miisscn.

Im Folgenden füge ich dicsen Deutungen unscres eigenthiimlichen Muskels eine weitere bei, von der ich glaube, dass sie die einfachste und plausibelstc ist, die übrigens andere nicht ausschliesst, d. h. wenu auch meine Anschaumg das Richtige trifft, so kann doch del Crampton'sche Muskel immer noch die Wölbung der Hornlıaut verändern und den Schlemm'schen Canal öffnen.

Ich halte die drei Muskelfasergruppen: Tensor choreoideare: Crampton'scher Mnskel und Miiller'sche Portion fur einen Accomodationsapparat, der, bedeutend kräftiger gestaltet als im Sängethierange, doch im Wesentlichen diesclbe Function ansiibt, die hier iter Tensor' choreoideae allein besorgt.

1 Het Accomodatievelmogen der (logen, 1853, pag. 75.

2 Ebendil, pag. 8?.

S. C. C. 
Um diese Anschanung zu begriinden, muss ich anf einige Eigenthïmlichkeiten des Vogelauges näher eingehen.

Znuächst existirt bei Vögeln (ob es hier Ausnahmen gibt, muss dahingestellt bleiben) weder ein Canalis Petiti, noch eine Zonula Zinnii in dem Simne wie bei Sängethieraugen, vielmeh. ist, wie dies Fig. 2 und 3 zeigt, die Linsencapsel in grösserer Ausdehnung mit den Ciliarfortsätzen verwachsen. In Fig. 3 sieht man Fasern, welche, zwischen den Ciliarfortsätzen verlaufend, diese Verbindnng zu befestigen scheinen. Halbirt man das Auge eines eben getödteten Huhnes, so dass die ebenfalls halbirte Linse in situ bleibt, fasst dann diese und sueht sie ron den Cilialfortsätzen abzulueben, so gewahrt man, dass sich eher die Liuse aus ilırer Capsel löst, als letztere von den Ciliarfortsätzen. Es ist also bei den Vögeln die Linse nicht an einer Zonula Zimuii und einer Hyaloidea, sonderu an den Ciliarfortsätzen beziiglich am rordersten Theil der Choreoidea, aufgehängt.

Fiihrt man den genannten Zug an der Linse aus, so gewahrt man noch etwas Zweites. Man sieht nämlich, dass der ganze rordere Theil der Choreoidea sich nach der Axe des Auges hin bewegt, so dass der ausgedehnte Spaltraum, der sich zwischen Choreoidea und Sclera befindet, an Breite zunimmt. Es ist dieser ein Analogon des Fontan a'schen Ranmes; el erstreckt sich rom Ligamentum pectinatum bis an die Einpflanzungsstelle del Fasern des 'Tensor choreoideae. Dieser ganze Ranm, der insbesondere beim Uhu eine enorme Ausdelnung hat (Fig. 2 and 3 als leer gelassener Ranm ersichtlich), ist nur von spairlichen lockeren Fäserchen uberbrickt, so dass, abgesehen rom Lignmentum pectinatum, der vordere Theil der Choreoidea an dic Sclera und ilnen Adnexen nur als angelegt, nicht angewachsen betrachtet werden kamn. Er kamn mit Leichtigkeit kleine Ortsverändermngen g'egen die Axe des Anges hin ausfïhren.

Diese Bewegrnngen an den rorderen Theilen der Choreoidea, welche man, wie gesagt, mit Leichtigkeit an jerlem friselsen odel gehärteten Ange herrorrufen kann, sind offenbar dieselben, welche die Choreoidea in Leben ansfihlen muss, soll sich der Durelymesser der Linse vergrössem oder verkleinern, wic das bei der Accomodation der Fall ist. Der Zug, den miln ansibt, weml man die Linse ron den Ciliarfortsiatzen abzulueben sucht, entspricht 
offenbar dem Zuge, den die Linse selbst auf die Ciliarfortsätze und die Choreoidea ausiibt, wenn sie sich stärker zu wölben sucht.

Es fungirt also dieser bewegliche Theil dcr Choreoidea als ein Aufhängcband der Linse ähnlich dem Theile der Membrana hyaloidea des Säugethieranges, welcher dic hintere Wand des Canalis Petiti bildet. Nun hat die Linse des Vogelanges abcr noeh ein zwcites, in der Functionsweise der Zonula Zinnii entsprechendes Aufhängeband; es ist dies das ungemein mächtige Ligamentum pectinatum. (Fig. 2 und 3 L. p.) 广̈bt man den oben beschriebenen Zug an einer Linse aus, so sieht man, wie sich die Fasern dieses Ligamentes gleichzeitig mit der Bewegung der Choreoidea auspannen. Beim Uhu haben dicsc Fasern eine Länge von cirea $4 \mathrm{Mm}$. nnd machen eine Täuschung iiber diescs Verhalten unmöglieh.

Dass das Ligamentum pectinatum der Vögel als Aufhäng’eband der Linse wirkt, muss an eh zur Erklärung der Accommodation, welehe blos durch den Tensor choreoidea hervorgerufen wird, angenommen wcrden. Denn wenn die Versuehc, welche uns sagen, dass der intraoculare Druck in der Vorderkammer und im Glaskörper merklich gleich ist, ${ }^{1}$ richtig sind, und wenn es weiter richtig ist, dass die Linse im Ruhczustande des Auges flacher ist, als ihrer Gleichgewichtslage entspricht, so muss es das Ligamentum peetinatum sein, welches sie hindcrt, ihre Gleielgewichtslage cinzunehmen. In der Choreoidea findet sie bei der Configuration des Vogelauges kein irgend nennenswerthes Hinderniss. Wäre abcr der Druck in der Vorderkammer niedriger als im Glaskörper, so würde die Linse bei der Contraction des Tensor choreoideae zwar nach rome riicken, aber für die bei der Accommodation thatsäehlieh nachgewicsene Krïmmungszunalume ihrer Flächen wäre kein zulänglicher Grund vorhanden.

Es lässt sich naeh dem Mitgetheilten nun mit Lcichtigkeit zeigen, dass der in Rede stehende Muskelapparat bei seiner Action die Wölbungen der Linse erhöhen muss, sowie dies im Säugetlicerange der Fall ist, wenn man voraussetzt, dass hier und dort dic elastischen Krifte der Aufhängehiinder es sind, welche

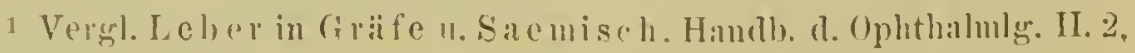
nag. 368. 
im Ruhezustande des Aug'es die Linse abflachen. Man kann nun aber in diesem Punkte einer anderen Ansicht sein. Man kann es für unwahrscheinlich halten, dass die Zonula Zinnii ein ganzes Leben lang fast immer (im Alter continuirlich), über ihre Gleichgewichtslage gedehnt sei, und rom Sängethierauge sag'en, del intraoculäre Druck im Glaskörper sei es, der Choreoidea und Hyaloidea anspamne. Auch auf diese Weise kann ein Zug auf die Linse ansgetibt werden, den dieselbe abzuplatten geeignet ist, und für welchen die Hyaloidea, beziiglich Zonula Zinnii nur als iibertragendes Band wirkt.

In dieser Beziehung muss ich, soll diese Anschanung auf das Vogelauge iibertragen werden, noch auf folgenden Umstand aufmerksam machen.

Wird das Ligamentum pectinatum als für Flìssigkeiten undurchgängig ang'enommen, so kann der Druck des Kammerwassers durch dieses Band in derselben Weise einen Zng auf die Linse ausiiben, wie dies der Druck des Glaskörper's durch die Choreoidea thut. Nach den Untersuchungen von Königrstein ${ }^{1}$ ist in der That beim Säugethicrauge die Vorderkammer durcl eine zarte Epithelialmembran, welche zwischen den Fasel'n des Ligamentum peetinatum ausgespannt ist, rom Fontana'schen Raum abgesperrt; Ähnliches liesse sich anch beim Vogelange vermuthen.

Da uiber diesen Punkt aber nieht alle Antoren einig 'zn sein scheinen, und Leuckart geradezu annimmt, dass bein Vogel unter gewissen Umständen das Kammerwasser durch das Ligamentnm pectinatum in den Foutana'sehen Raum einströne, ${ }^{2}$ so mögren diese Verhältnisse hier nur erwähnt sein. Icl will später zeigen, dass meine Auschaunng von der Accommodation des Vogelauges unabhängig davon ist, welcher der beiden Fiille zutrifft, ob die Linse im Rulıezustand des Anges ihren äquatorialen Zng num den elastisehen Kraften ihrer Anfhängebäuder verdankt, oder ob derselbe dem intraoculairen Drucke entspringt." Zunächst aber will ich voraussetzen, dass das Letztere der Fall ist, und dass die Vorderkammer in Ligamentum pectinatum einen Abschlıss

1 Vergl. (ir it fe's Areh. i. Ophthalmolog. XXV. 3, pag. 28!).

L. c. pag. 235. 
findet, so dass der hier herrschende hydrostatisehe Druck mithilft, die Linse abzuflachen.

Die Wirkung'sweise der fragliehen Muskeln des Vogelauges ergibt sich nun leicht ans der beistehenden schematischen Zeichnung.

$L$ ist die Linse, welche nahe ihrem Äquator dureh die zwei Aufhäng'ebänder gehalten wird, deren vorderes das Ligamentum pectinatum, deren hinteres die Choreoidea ist. Auf beide wirkt der intraveuläre Druek in der Riehtung der Pfeile of und rieht den Äquator uiber seine Gleichgewi.htslage naeh aussen. $S S$ ist die Sclera und der feste äussere Antheil der Cornea, $C$ die innere Lage der Cornea, die, wie oben nnitgetheilt. bis zu einem gewissen Grade verschiebbar ist, und speciell an ihrem Ende aus verhältnissmässig lockerem, dehnbarem Gewebe besteht (Fig. 3).

Es leuchtet nun ein, dass jede Muskelaction, welche die Distanz

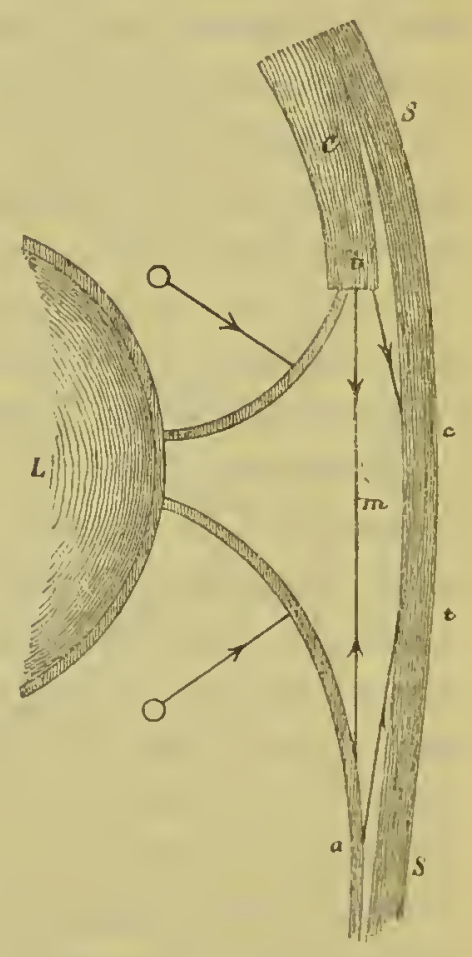
zwisehen dem Punkte a der Choreoidea und dem Punkte b der Cornea verringert den von den Aufhänģebändern der Linse ansgeiibten Zng verkleinern und es also dieser ermöglichen muss, ilırer Gleichgewichtsform näher zu riieken. Alle drei Mnskelportionen num bewirken diese Verringermng der genannten Distanz. Der 'Tensor choreoideac (at), der an der Sclera entspringt und bei " endet, riickt den letzteren Punkt nach vorne, der Musmulus Crmmploniamus (br), der anch an der Selera entspringt nud an der inneren Hornhautlamelle endet, riickt $b$ nach riickwäits und die M ül l er'sehe Portion (m) ist geradezu zwischen den beiden Punkten (" und b) ansgespannt.

Beim Uhu (Fig. 1 und 2) hat jeder der beiden sehematischen Punkte " nnd b seinen besonderen Mnskel — eine Miiller'sche 
Portion gibt es hier nicht - und ich glaube, man sieht unmittelbar aus der Zeichuung (Fig. 2), dass der Crampton'sche Muskel das Ligamentum pectinatum abspamnen muss. Noch viel klarer wird das am mikroskopischen Präparat, an welchem das eigenthümliche lockere Gefüge des Thiciles der Cornea, an welchem sich sowohl der Muskel, als das Ligament ansetzt, die Delinbarkeit desselben unmittelbar anschaulich macht.

Bei der Taube (Fig. 3) liegen die Dinge so, wie in unserem Schema. Nur setzen sich einige Muskelfasern nicht direct an ihrem Bestimmungsort an, sondern sind durch Sehnen mit ilum verbunden. So das vordere. Ende der Minller'schen Portion und ein Theil der Faseru des Crampton'schen Muskels, welche durch zwei Sehnenbiundel, dic den Schlemm'schen Canal zwischen sich fassen, in das dehmbare Hornhantgewebe ubbergehen.

Ich will noch hervorhcben, dass verschiedene Schnitte durch ein Vogelange und noch mehr Sclnitte durch verschiedene Vogrelaugen den in Rede stehenden Mnskelapparat in mannigfaltig'en Modificationen zeigen, wie dies iibrigens oftmals beschrieben wurde. Zwei möglichst differente Typen, den des Uhu und der Taube, liabe ich meinen Betrachtungen zu Grunde gelegt; es ist mir aber keine Modification bekannt geworden, welche sich nicht ohne Weiteres der dargelegten Anschaumng unterordnet.

Ist num das Ligamentum pectinatnm für das Kammerwasser durchäangig, so wird hicedureh der anseinandergesetztc Accommodationsmechanismus nicht becinflusst. Denn denken wir uns in

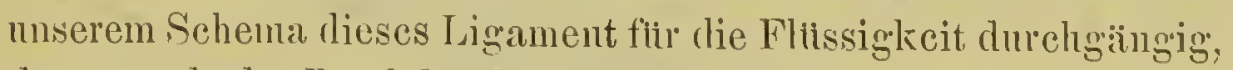
dann wiirde der Rand der Linse roranssichtlich so weit nach innen und hinten riicken, bis das Liganent wieder angesplannt ist. Auch danı noch wiirde die Zusammenziehung unserer Muskech denselben qualitativen Effect haben wic frilher.

Es könnte weiterhin Jemand die Voraussetyutg machen, dass der Druck im Kammerwasser ein geringerer ist als im Glaskörper. Es misste dann, wie schon hervorgehoben, die Differenz, welche \%wischen dem auf die vordere mo dem anf dic hintere Linsenfliche wirkenden Drucke herrscht ron der Choreoiden getragen 
werden, und die Aeeomorlation wiirde wenigstens in erster Linie nicht mehr auf einer Formverändernng, 'sondern anf einer Lageveränderurg der Linse beruhen. Die Formveränderung wiirde sehon desshalb nieht melı in Betraeht kommen, weil im Ruhezustand des Auges kein Zug in der Riehtung des äquatorialen Linsenradius, sondern nur ein soleher naeh linten auf die Linse wirken wiirde.

Aus dem Umstande aber, dass Cramer ${ }^{1}$ die Zunahme der Linsenwölbung bei elektriseher Reizung des Vogelauges beobachtet hat, sowie mit Riieksieht anf die oben angefiihrten Messungen intraoeulären Druekes kann diese Voranssetzung wohl als nugereehtfertigt zuriiekgewiesen werden. Sie miisste das anel, wenn der Tensor ehoreoideae allein in der gangbaren Weise zur Erklärnng der Aeeomodation herange\%ogen werden soll.

Es ist also die Linse des Vogelauges nahe ihrem Äquator an der Choreoidea einerseits, dem Ligamentum peetinatum andererseits aufgehängt. Im aeeommorationslosen Zustande des Auges wird sie dureh einen radiären Zug, welehen diese Aufhängebänder ansiiben, abgeplattet, analog dem Zuge, den die Membrana hyaloidea nnd die Zonnla Zinnii im Säugethierauge ansiiben. Sei es uun, dass dieser Zug in den elastisehen Kräften der Aufhängebänder, sei es, dass er im intraoeulären Druek seinen Ursprung hat, immer dient der in Rede stehende Muskelapparat des Vogelanges dazın, die Anfhängebänder abzuspannen und so zu elmögliehen, dass die Linse sieh ihrer Gleichgewielıtslage nähere.

Diese Ansehaung findet in einem alten Versuehe Cran er's ihre Bestätigung, ${ }^{2}$ weleher zeigte, dass die Linse des Vogelauges ihre Aecommodationsbewegung einstellt, wenn die Iris abgetrennt wird. Naeh den Dargelegten landelt es sieh hier nieht um die Iris als soleher, sondern 1 m das Ligamentum peetinatum, das natiirlieh bei dieser Abtremmug durelırissen wird. Es ist damı eben der ganze Auflä̆nge:ıpparat der Linse zerstört. und es kann von einem Abspamnen desselben dureh Muskelaction nieht meh" die Rede sein.

\footnotetext{
1 I. (. pag. 9 ).

2 L. c. pag. 90.
} 
S. Fixner: Funclion d. Musc. Cramplonianus.

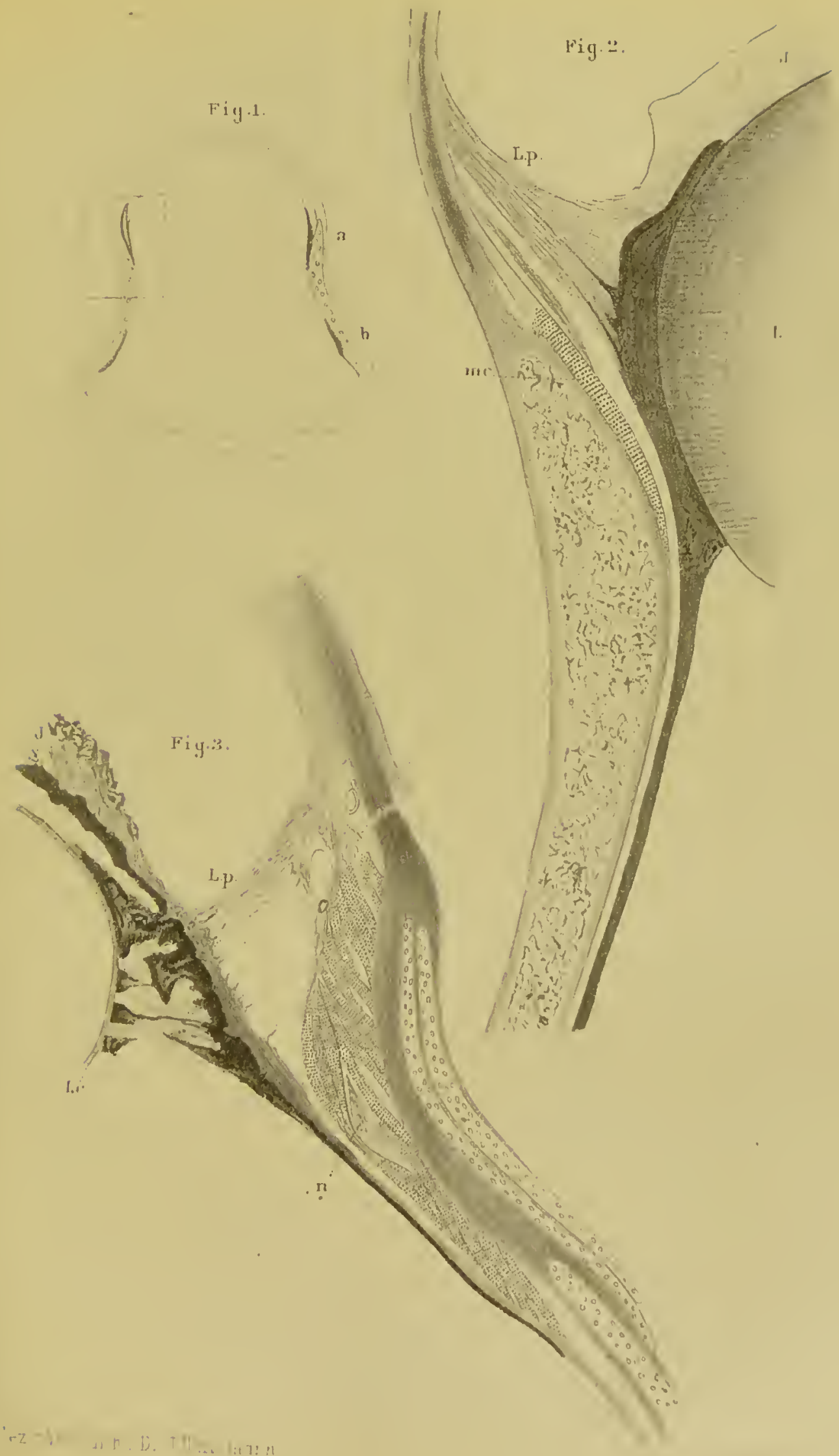

Ki:

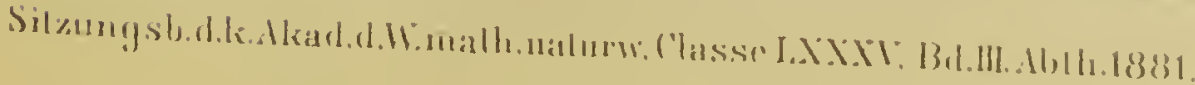





\section{Erklärung der Tafel.}

Fig. 1. Durchschnitt durch das Auge eines Uhu nach Brücke. Bei $a$ ist der Musculus Cramptoniams, bei $b$ der Tensor choreoideae. Die durch ein Rechteck eingerahmte Stelle ist in

n 2 nach einem anatomischen Präparat bei Lupenvergrösserung wiedergegeben, doch ist der Uvealtractus und die Linse $L$ noch hinzugefügt. Der Crampton'sche Muskel $m c$ ist durch Punktirung: hervorgehoben. $J$ Iris; $L p$ Ligamentum pectinatum.

3. Die drei Muskelfasergruppen einer Taube. Auch hier sind die Muskelfasern punctirt. $J$ Iris; $L c$ Linsenkapsel; $L p$ Ligamentum pectinatum; $n$ ein Nervenstamm, der sich zwischen die beideu Bäuche der Müll er'schen Portion einschiebt. 


$$
1
$$

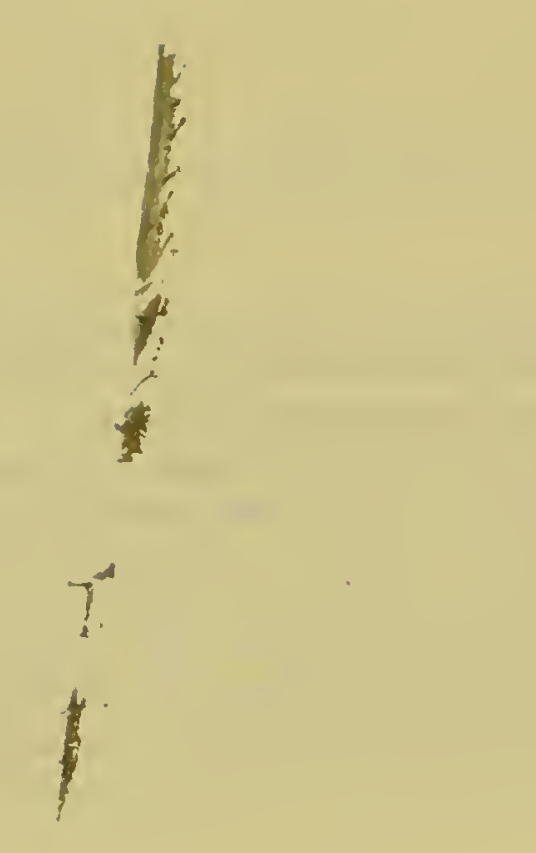

